\title{
Erratum to: Maintenance of bladder urothelia integrity and successful urothelialization of various tissue-engineered mesenchymes in vitro
}

\author{
Sara Bouhout $^{1,2} \cdot$ Julie Tremblay $^{3} \cdot$ Stephane Bolduc $^{2}$
}

Published online: 1 October 2015

(C) The Society for In Vitro Biology 2015

Erratum to: In Vitro Cell.Dev.Biol.-Animal

DOI 10.1007/s11626-015-9923-x

The metadata listing the Given Names and Family Names is wrong. The correct order is Sara Bouhout, Julie Tremblay, and Stephane Bolduc.

The online version of the original article can be found at http://dx.doi.org/ 10.1007/s11626-015-9923-x.

Sara Bouhout

sara.ucergy@hotmail.fr

1 Centre de recherche en organogénèse expérimentale de l'Université, Laval/LOEX Faculté de médecine, Université Laval,

Québec, Canada

2 Centre de recherche en organogénèse expérimentale de l'Université, Laval/LOEX, Faculté de médecine, Centre de Recherche du CHU de Québec, Université Laval, Québec, Canada

3 Service d'anatomopathologie du CHU de Québec, Québec, Canada 